\title{
Stochastic Model Based On Order Statistics for Estimation of Expected Time to Reach the Breakdown Point
}

\author{
Dr. Mrs. T. Chitrakala rani ${ }^{1}$, Mrs. .C.Sukumari ${ }^{2}$ \\ ${ }^{1}$ Associate professor in Mathematics, K.N.G.Arts College for women (Autonomous), Thanjavur, India. \\ ${ }^{2}$ Associate professor in Mathematics, Ponnaiyah Ramajayam college of Engg and Tech, Thanjavur, India.
}

\begin{abstract}
In an automobile system repairs and replacements is a task which requires careful and detailed monitoring. To estimate the expected time to reach the breakdown point(Total failure state) and variance of a system due to the successive occurrence of small reparable break downs in $(0, t]$ by using the applications of Order statistics, and assuming that the threshold follows gamma distribution. The effects of increasing in the length of inter arrival times between small reparable break downs on the values of mean and variance has been found. The analytical results are substantiated with numerical illustrations.
\end{abstract}

Key words: Breakdown point, Gamma distribution, Order statistics.

\section{Introduction:}

The life span of systems that fail or the duration of time a deteriorating system works with the required amount of efficiency, or the amount of time an idle system stays in proper condition for usages, are usually probabilistic. Hence we cannot predict with certainty when the systems will fail or become unable to function with required level of efficiency. Upon failure, the system is either repaired or replaced by a new and identical one. It is generally assumed that after repair, the system will return to as good as new state (complete repair) or the state just prior to failure (minimal repair). In practice, the repair of a failure system will usually yield a functioning system; its successive survival times are decreasing and finally dying out. For deteriorating systems, this is often the case. A system which is exposed to the cumulative damage process due to the occurrence of small reparable break down is considered.

Prochan (1973) studied some models for the life distribution of a device subject to a sequence of shocks occurring randomly in times events in a Poisson process. Sathiyamoorthi (1998) determined the expected time for recruitment by using shock model. Esary J.D (1973) discussed that any component or device when exposed to shock which cause damage to the device or system is likely to fail when the total accumulated damage determines the life time of the component or device. Ramanarayanan.R(1976) considered a device which is exposed to the cumulative damage process due to the occurrence of shocks .Ganeshan.M.S, and Chitra kala rani.T (2002) makes an attempt to examine the economic threshold level by keeping industrial accidents in view, also finds that the expected time to reach the uneconomic status of the organization and its variance by using cumulative damage model. Ji Hwan cha, JieMi (2011) derived a stochastic survival model for a system under random shock process which affects the survival of the system in a complicated way. They derived the lifetime distribution of the system, and investigated the effect of environmental factors on the failure process of the system.

In this paper the expected time to reach the breakdown point(Total failure state) and its variance by assuming threshold follows gamma distribution. Also the concept of order statistics is used to represent the distribution of interarival times between successive reparable breakdown epochs. Since the inter arrival times between small reparable break down epochs are assumed to be identically independent random variables, they can be arranged as a monotonic increasing sequence, thereby giving rise to order statistics. The expected time to reach the Breakdown point(Total failure state) and its variance are obtained taking the distribution of interarrival times as first order (minimum) and $\mathrm{n}^{\text {th }}$ order (maximum) statistics respectively.

\section{Assumption of the Model:}

(i) Small reparable Breakdowns occur at k-random epoch and at every epoch it causes damage to the system in terms of machine hours

(ii) The system fails when the total accumulated damage exceeds a random threshold.

(iii) The inter arrival times between successive occurrence of damage identically independent random variables

(iv) The process which generates the small reparable break downs, the sequence of damages $X_{i}$ and a threshold level $\mathrm{Y}$ which is itself a random variable are independent. 


\subsection{Notations:}

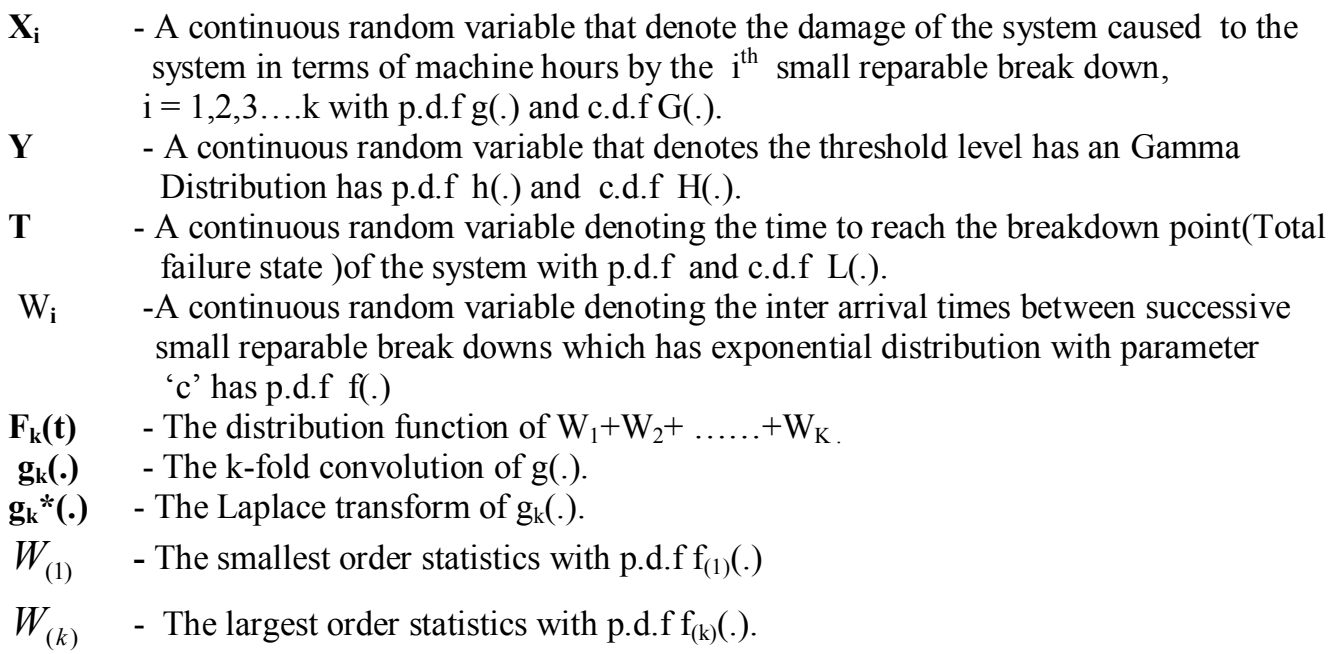

\subsection{Results:}

The gamma distribution is frequently used to model in life testing. Two parameter gamma distributions is most popular distribution for analyzing life time data. Let $\mathrm{Y}$ be the random variable which follows gamma distribution $\mathrm{Y} \sim \mathrm{G}(\mathrm{n}, \lambda)$.

$$
\begin{gathered}
P\left[\sum X_{i}<Y\right]=\int_{0}^{\infty} g_{k}(x) \overline{H(x)} \\
=\int_{0}^{\infty} g_{k}(x)\left[\sum_{i=0}^{n-1} \frac{e^{-\lambda x}(\lambda x)^{i}}{\Gamma_{i}}\right] d x \\
P\left[\sum X_{i}<Y\right]=\sum_{i=0}^{n-1}\left[\frac{b \lambda^{i}}{(b+\lambda)^{i+1}}\right]^{k}
\end{gathered}
$$

The survival function $s(t)$ which is the probability that an individual survives for a time ' $t$ '.

$\mathrm{S}(\mathrm{t})=\mathrm{P}(\mathrm{T}>\mathrm{t})=$ Probability that the system survives beyond ' $\mathrm{t}$ '

$=\sum_{k=0}^{\infty} \Gamma[$ There are exactly $k$ ins $\tan t \sin (0, t)] \times \Gamma($ the system doesnot fail in $(o, t])$.

By renewal theory

$\mathrm{P}($ There are exactly $k$ ins $\tan t \sin (0, t])=F_{k}(t)-F_{k+1}(t) \quad$ with $F_{0}(t)=1$

$$
\begin{aligned}
& \mathrm{S}(\mathrm{t})=\sum_{k=0}^{\infty}\left[F_{k}(t)-F_{k+1}(t)\right] \sum_{i=0}^{n-1}\left[\frac{b \lambda^{i}}{(b+\lambda)^{i+1}}\right]^{k} \ldots . \\
& \mathrm{L}(\mathrm{T})=1-\mathrm{S}(\mathrm{t}) . \\
& \mathrm{L}(\mathrm{T})=\sum_{i=0}^{n-1}\left[1-\frac{b \lambda^{i}}{(b+\lambda)^{i+1}}\right] \sum_{k=1}^{\infty} F_{k}(t)\left[\frac{b \lambda^{i}}{(b+\lambda)^{i+1}}\right]^{k-1}
\end{aligned}
$$

and Laplace transform of $\mathrm{L}(\mathrm{t})$ is obtained as

$$
\mathrm{L}^{*}(\mathrm{~s})=\sum_{i=0}^{n-1} \frac{\left[1-\frac{b \lambda^{i}}{(b+\lambda)^{i+1}}\right] \mathrm{f} *(\mathrm{~s})}{1-\frac{b \lambda^{i}}{(b+\lambda)^{i+1}} \mathrm{f} *(\mathrm{~s})}
$$




$$
L^{*}(s)=\sum_{i=0}^{n-1} \frac{\left[1-\frac{b \lambda^{i}}{(b+\lambda)^{i+1}}\right][c / c+s]}{\left[1-\frac{b \lambda^{i}}{(b+\lambda)^{i+1}}(c / c+s)\right]},
$$

2.2.1: Case (i): The inter arrival are i.i.d .f(.) $\exp (\mathrm{c})$.

$$
\begin{aligned}
& \therefore \text { Mean }=\mathrm{E}(\mathrm{T})=\frac{1}{c} \sum_{i=0}^{n-1} \frac{(b+\lambda)^{i+1}}{(b+\lambda)^{i+1}-b \lambda^{i}} \\
& \text { Variance }=\mathrm{V}(\mathrm{T})=\left[\frac{2}{c^{2}} \sum_{i=0}^{n-1}\left[\frac{(b+\lambda)^{(i+1)}}{\left((b+\lambda)^{i+1}-b \lambda^{i}\right)}\right]^{2}\right]-\left[\frac{1}{c} \sum_{i=0}^{n-1} \frac{(b+\lambda)^{(i+1)}}{\left((b+\lambda)^{i+1}-b \lambda^{i}\right)}\right]^{2}
\end{aligned}
$$

This is the result in the most general form of the inter arrival times of small reparable break down epochs where $\mathrm{W}_{\mathrm{i}}$ 's are i.i.d. $\mathrm{W}_{1}, \mathrm{~W}_{2} \ldots \ldots . \mathrm{W}_{\mathrm{K}}$ be arranged in increasing order of magnitude so that we have the sequence as $\mathrm{W}_{(1)}, \mathrm{W}_{(2)}, \mathrm{W}_{(3)}, \ldots \ldots \mathrm{W}_{(\mathrm{k})}$. Here $\mathrm{r}=1,2, \ldots . \mathrm{k}$ are called the order statistics and with $\mathrm{W}_{(1)}$ as the first order statistic (smallest) and $\mathrm{W}_{(\mathrm{k})}$ the $\mathrm{k}^{\mathrm{th}}$ order statistic(larest) and the random variables $\mathrm{W}_{(1)}, \mathrm{W}_{(2)}, \mathrm{W}_{(3)}, \ldots \ldots \mathrm{W}_{(\mathrm{k})}$ are not independent. The p.d.f of " $r$ 'th' order statistic is given by

$$
f_{w(r)}(t)=r\left(\begin{array}{l}
k \\
r
\end{array}\right)[F(t)]^{r-1} f(t)[1-F(t)]^{k-r}
$$

2.2.2: Case ii : For order statistics $\mathrm{W}_{(1)}$

Now let $f_{w(1)}^{*}(s)$ be the laplace transform of $f_{W_{(1)}}(t)$,

$$
\begin{aligned}
& \text { If } f_{W_{(1)}}(t)=\mathrm{k}[1-\mathrm{F}(\mathrm{t})]^{\mathrm{k}-1} \mathrm{f}(\mathrm{t})=\mathrm{k}\left(\mathrm{e}^{-\mathrm{cx}}\right)^{\mathrm{k}-1} \mathrm{ce}^{-\mathrm{cx}} \\
& f_{\left.W_{(1)}\right)}^{*}(s)=\frac{k c}{s+k c}
\end{aligned}
$$

Substituting in (2) we get

$$
\mathrm{L}^{*}(\mathrm{~s})=\sum_{i=0}^{n-1} \frac{\left[1-\frac{b \lambda^{i}}{(b+\lambda)^{i+1}}\right] \frac{k c}{s+k c}}{1-\frac{b \lambda^{i}}{(b+\lambda)^{i+1}} \frac{k c}{s+k c}}
$$

on simplification we get

$$
=\sum_{i=0}^{n-1} \frac{k c(b+\lambda)^{i+1}-k c b \lambda^{i}}{(s+k c)(b+\lambda)^{i+1}-k c b \lambda^{i}}
$$

Now to find $\mathrm{E}(\mathrm{T})$ we take $E(T)=\left\langle\left.\frac{-d L^{*}(s)}{d s}\right|_{s=0}\right.$

$$
\begin{aligned}
E(T) & =\frac{1}{k c} \sum_{i=0}^{n-1} \frac{(b+\lambda)^{(i+1)}}{\left[(b+\lambda)^{(i+1)}-b \lambda^{i}\right]} \\
V(T) & =\frac{2}{k^{2} c^{2}} \sum_{i=0}^{n-1}\left[\frac{(b+\lambda)^{(i+1)}}{(b+\lambda)^{(i+1)}-b \lambda^{i}}\right]^{2}-\left[\frac{1}{k c} \sum_{i=0}^{n-1} \frac{(b+\lambda)^{(i+1)}}{\left[(b+\lambda)^{(i+1)}-b \lambda^{i}\right]}\right]^{2}
\end{aligned}
$$

2.2.3: Case iii: For order statistics $\mathrm{W}_{(\mathrm{k})}$

Let us take the p.d.f of the $\mathrm{k}^{\text {th }}$ order statistics $\mathrm{W}_{(\mathrm{k})}$

And $f_{W(k)}(t)=\mathrm{k}\left(1-\mathrm{e}^{-\mathrm{cx}}\right)^{\mathrm{k}-1} \mathrm{ce}^{-\mathrm{cx}}$ 


$$
f_{W(k)}^{*}(s)=\frac{k ! c^{k}}{(c+s)(2 c+s) \ldots \ldots(k c+s)}
$$

Substituting in (2) we get

$$
\mathrm{L}^{*}(\mathrm{~s})=\sum_{i=0}^{n-1} \frac{\left[1-\frac{b \lambda^{i}}{(b+\lambda)^{i+1}}\right] \frac{k ! c^{k}}{1-\frac{b \lambda^{i}}{(b+\lambda)^{i+1}} \frac{k ! c^{k}}{(c+s)(2 c+s) \ldots \ldots(k c+s)}}}{1(2 c+s) \ldots \ldots(k c+s)}
$$

Let $\mathrm{A}=(c+s)(2 c+s) \ldots \ldots(k c+s)$

$\mathrm{L}^{*}(\mathrm{~s})=\sum_{i=0}^{n-1} \frac{\left[1-\frac{b \lambda^{i}}{(b+\lambda)^{i+1}}\right] \frac{k ! c^{k}}{A}}{1-\frac{b \lambda^{i}}{(b+\lambda)^{i+1}} \frac{k ! c^{k}}{A}}=\sum_{i=0}^{n-1} \frac{k ! c^{k}\left[(b+\lambda)^{i+1}-b \lambda^{i}\right]}{A(b+\lambda)^{i+1}-b \lambda^{i} k ! c^{k}}$

Now $E(T)=\left\langle\left.\frac{-d L^{*}(s)}{d s}\right|_{s=0}\right.$

$$
\begin{aligned}
& \left\langle\frac { - d A } { d s } | _ { s = 0 } = c ^ { k - 1 } k ! \sum _ { j = 1 } ^ { k } \frac { 1 } { j } \text { and } \left\langle\left.\frac{d^{2} A}{d s^{2}}\right|_{s=0}=c^{k-2} k !\left[\left(\sum_{j=1}^{k} \frac{1}{j}\right)^{2}-\sum_{j=1}^{k} \frac{1}{j^{2}}\right]\right.\right. \\
& E(T)=\frac{1}{c} \sum_{i=0}^{n-1} \frac{(b+\lambda)^{(i+1)} \sum_{j=1}^{k} \frac{1}{j}}{\left.(b+\lambda)^{(i+1)}-b \lambda^{i}\right]}
\end{aligned}
$$

$V(T)=\frac{1}{c^{2}} \sum_{i=0}^{n-1}\left[\frac{(b+\lambda)^{(i+1)}\left(\sum_{j=1}^{k} \frac{1}{j^{2}}-\left(\sum_{j=1}^{k} \frac{1}{j}\right)^{2}\right)}{\left[(b+\lambda)^{(i+1)}-b \lambda^{i}\right]}+\frac{2\left((b+\lambda)^{(i+1)} \sum_{j=1}^{k} \frac{1}{j}\right)^{2}}{\left[(b+\lambda)^{(i+1)}-b \lambda^{i}\right]^{2}}\right]-\left[\frac{1}{c} \sum_{i=0}^{n-1} \frac{(b+\lambda)^{(i+1)} \sum_{j=1}^{k} \frac{1}{j}}{\left.(b+\lambda)^{(i+1)}-b \lambda^{i}\right]}\right]^{2}$

\subsection{Numerical Illustration:}

2.3.1:Case (i): Threshold follows gamma distribution $\sim \mathrm{G}(\mathrm{n}, \lambda)$.

$$
\begin{aligned}
E(T) & =\frac{1}{c} \sum_{i=0}^{n-1} \frac{(b+\lambda)^{(i+1)}}{\left[(b+\lambda)^{(i+1)}-b \lambda^{i}\right]} \\
V(T) & =\left[\frac{2}{c^{2}} \sum_{i=0}^{n-1}\left[\frac{(b+\lambda)^{(i+1)}}{\left((b+\lambda)^{i+1}-b \lambda^{i}\right)}\right]^{2}\right]-\left[\frac{1}{c} \sum_{i=0}^{n-1} \frac{(b+\lambda)^{(i+1)}}{\left((b+\lambda)^{i+1}-b \lambda^{i}\right)}\right]^{2}
\end{aligned}
$$

We find out the values of $\mathrm{E}(\mathrm{T})$ and $\mathrm{V}(\mathrm{T})$ fixed values of the parameters $\lambda=0.4, \mathrm{~b}=0.2$ and $\mathrm{c}=1,2, \ldots 5, \mathrm{n}=2,4$ 
Table $1.1 \quad \mathrm{G} \sim \mathrm{G}(\mathrm{n}, \lambda)$ then $\mathrm{E}(\mathrm{T}) \& \mathrm{~V}(\mathrm{~T})$

\begin{tabular}{|c|l|l|l|l|}
\hline \multirow{2}{*}{$\mathrm{c}^{\mathrm{n}}$} & \multicolumn{2}{|c|}{$\mathrm{E}(\mathrm{T})$} & \multicolumn{2}{c|}{$\mathrm{V}(\mathrm{T})$} \\
\cline { 2 - 5 } & $\mathrm{n}=2$ & $\mathrm{n}=4$ & $\mathrm{n}=2$ & $\mathrm{n}=4$ \\
\hline 1 & 2.786 & 5.069 & 7.76 & 25.697 \\
\hline 2 & 1.393 & 2.535 & 1.94 & 6.424 \\
\hline 3 & 0.929 & 1.69 & 0.862 & 2.855 \\
\hline 4 & 0.696 & 1.267 & 0.485 & 1.606 \\
\hline 5 & 0.555 & 1.014 & 0.31 & 1.028 \\
\hline
\end{tabular}

2.3.2: Case (ii) : For the order statistics $\mathrm{W}_{(1)}$

$$
\begin{aligned}
& E(T)=\frac{1}{k c} \sum_{i=0}^{n-1} \frac{(b+\lambda)^{(i+1)}}{\left[(b+\lambda)^{(i+1)}-b \lambda^{i}\right]} \\
& \left.V(T)=\frac{2}{k^{2} c^{2}} \sum_{i=0}^{n-1}\left[\frac{(b+\lambda)^{(i+1)}}{(b+\lambda)^{(i+1)}-b \lambda^{i}}\right]^{2}-\left[\frac{1}{k c} \sum_{i=0}^{n-1} \frac{(b+\lambda)^{(i+1)}}{\left[(b+\lambda)^{(i+1)}-b \lambda^{i}\right.}\right]\right]^{2}
\end{aligned}
$$

Now $k=$ number of decision epochs. We find out the values of $E(T)$ and $V(T)$ fixed values of the parameters $\lambda=0.4, \mathrm{~b}=0.2, \mathrm{c}=1.5$ and $\mathrm{k}=1,2, \ldots .5, \mathrm{n}=2$

Table 1.2: For the order statistics $\mathbf{W}_{(1)}$

\begin{tabular}{|l|l|l|}
\hline $\mathrm{k}$ & $\mathrm{E}(\mathrm{T})$ & $\mathrm{V}(\mathrm{T})$ \\
\hline 1 & 0.8553 & 0.0209 \\
\hline 2 & 0.4276 & 0.0053 \\
\hline 3 & 0.2851 & 0.0023 \\
\hline 4 & 0.2138 & 0.0014 \\
\hline 5 & 0.1711 & 0.00082 \\
\hline
\end{tabular}

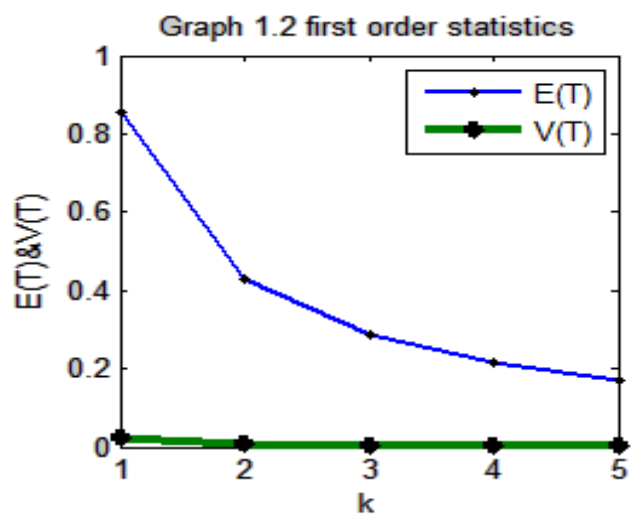

2.3.3: Case iii.:For the order statistics $\mathrm{W}_{(\mathrm{k})}$ :

$$
\begin{aligned}
& E(T)=\frac{1}{c} \sum_{i=0}^{n-1} \frac{(b+\lambda)^{(i+1)} \sum_{j=1}^{k} \frac{1}{j}}{\left.(b+\lambda)^{(i+1)}-b \lambda^{i}\right]}, \\
& \left.V(T)=\frac{1}{c^{2}} \sum_{i=0}^{n-1}\left[\frac{(b+\lambda)^{(i+1)}\left(\sum_{j=1}^{k} \frac{1}{j^{2}}-\left(\sum_{j=1}^{k} \frac{1}{j}\right)^{2}\right)}{\left[(b+\lambda)^{(i+1)}-b \lambda^{i}\right]}+\frac{2\left((b+\lambda)^{(i+1)} \sum_{j=1}^{k} \frac{1}{j}\right)^{2}}{\left[(b+\lambda)^{(i+1)}-b \lambda^{i}\right]^{2}}\right]-\left[\frac{1}{c} \sum_{i=0}^{n-1}\left[(b+\lambda)^{(i+1)}-b \lambda^{i}\right]\right]_{j=1}^{(b+\lambda)^{(i+1)}} \sum^{k} \frac{1}{[(b)}\right]^{2}
\end{aligned}
$$


Table 1.3 : Forder statistics $W_{(k)}$

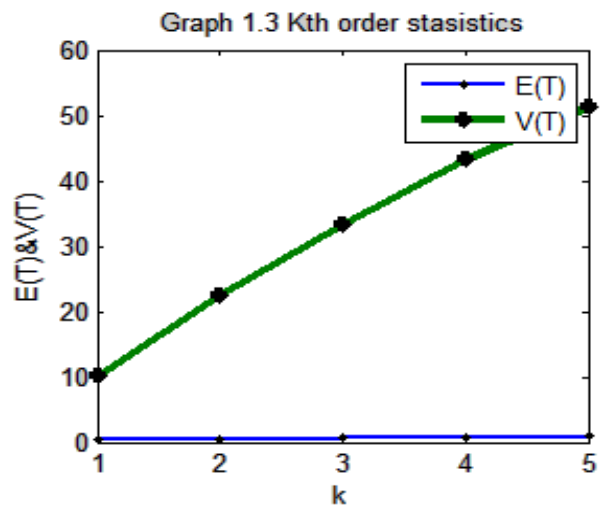

From Graph 1.1 when threshold follows gamma distribution which prolong the expected time to reach the breakdown point. From Graph 1.2 if the number of decision epochs in $(0, t)$ increases, then the length of interarrival times shorter.Hence $\mathrm{E}(\mathrm{T})$ and $\mathrm{V}(\mathrm{T})$ both become smaller.From Graph 1.3 if the number of decision epochs increases the $\mathrm{k}^{\text {th }}$ order statistics also increases, then the length of interarrival times increases.Hence $\mathrm{E}(\mathrm{T})$ and $\mathrm{V}(\mathrm{T})$ shows an increase.

\section{References}

[1] Esary .J.D, Marshall, A.W and Prochan.F (1973)“shock Models and wear processes”, Ann. of pro., Vol.12: pp-18-28

[2] Esary, J.D., Marshall, A.W. and Proschan F. (1973). Shock models and wear processes. "Ann. Probability”, 1(4), 627-649.

[3] Ramanarayanan.R (1976), “cumulative damage process and alertness of the worker", IEEE transaction on reliability, $R 25$ (4): pp281-83.

[4] Abhijit Dasgupta and Michael percht (1991) "Material Failure Mechanisms and Damage”, IEEE Transaction on Reliability, 40(5): pp-531-36.

[5] Gupta, R. D. and Kundu D. (1999). Generalized exponential distributions, Australian and New Zealand Journal of Statistics, 41, $173-188$.

[6] Ganeshan.M.S, Chitra kala rani.T (2002) "stochastic model for the cumulative effects of industrial accidents". A journal on stochastic process and its Applications, vol.5 (1); pp-53-57

[7] Ji Hwan cha,JieMi(2011) "On a stochastic survival model for a system under randomly variable environment" Methodology and computing in applied probability vol 13 (3); pp 549-561. 